(potentially a major application), and the conceptually difficult spectrum of phenomena ranging from straight electrolysis, through electrically assisted photolysis, to straight photolysis of water at electrically connected semiconducting electrodes without benefit of external batteries. (This whole field was initiated by a Japanese paper in Nature in 1972.) $\mathrm{TiO}_{2}$ and $\mathrm{SrTiO}_{3}$ have been tried, but other semiconductors with smaller bandgaps need to be developed, perhaps with dye sensitisers incorporated. The potential importance of this technology is very great.

In a beautifully lucid lecture G. Allen (Imperial College), basing himself on the Flory-Huggins insights, explained just why useful two-phase polymers can in general only be made by the stratagem of block copolymerisation, which alone permits the correct balance between the tendency to phase separate and the physical impossibility of doing so completely. To a metallurgist used to the interpretation of metallurgical phase diagrams in terms of the configurational entropy of crystalline solid solutions, the rationalisation of polymeric phase diagrams in terms of the configurational entropy of polymer chains was convincing. The laws of mixtures governing the physical properties of such systems were exemplified and the prospects for ungrafted solution-blended two-phase systems outlined. (Physically mixed polymer systems apparently have a Houdini-like proclivity for escaping from their most elaborate entanglements.) The formation of networks from T-shaped monomers was explained. This approach permits the design of tough structural plastics and rubbers, of materials with controlled water permeability and other special-function polyers. In this lecture the rationale of a lifetime's research strategy was made manifest.

The symposium was an unqualified success, and it is to be hoped that the SRC will feel encouraged to continue to sponsor such occasions.

\section{Green light for the signal hypothesis?}

\section{from Pamela Hamlyn}

THE work on secreted proteins, particularly those of the liver and pancreas, has demonstrated that these proteins are synthesised on ribosomes bound to endoplasmic reticulum, then transferred through the membrane into the lumen so that their synthesis and secretion form a continuous process. What

Pamela Hamlyn is at the MRC Laboratory of Molecular Biology, Cambridge. is much less certain is the way in which the messenger RNAs which code for secretory proteins are selected for translation by membrane-bound ribosomes. That the selection may be a consequence of a particular nucleotide sequence in these mRNAs, or due to the activity of a separate class of ribosomes, are considerations which have found some experimental support, but the suggestion for which there is most experimental evidence is that it is a characteristic of the protein itself (namely the amino acids found at the amino terminus of a precursor form of the mature protein) which leads to its synthesis by membrane-bound ribosomes. A model for the mechanism by which the ribosome is brought in contact with the membrane by way of the $\mathrm{N}$-terminal amino acids has been suggested by G. Blobel and his colleagues (for example, Blobel \& Dobberstein I. cell Biol. 67, 835; 1975).

Some very convincing evidence that it is the protein product, rather than the mRNA or ribosomes, which is responsible for the specificity of attachment of polysomes to membranes, has been produced by Harvey Lodish's group at the Massachusetts Institute of Technology (Wirth et al. Cell 10, 253; 1977). Working with Sindbis virusinfected chicken embryo fibroblasts, they have isolated a $26 \mathrm{~S}$ viral mRNA which codes for three proteins; one is a core protein which is eventually associated with the 42S RNA viral genome and the other two are glycoproteins required for the lipid bilayer which envelops the mature virus. The proteins are translated sequentially from one initiation site on the mRNA: first the core protein, then the two envelope proteins. This recent report is concerned with the localisation of the polysomes involved in the synthesis of these two classes of protein. It was found that $70 \%$ of the 26S RNA is bound to membrane and that newly synthesised core protein is localised on the cytoplasmic side of the endoplasmic neticulum and remains free in the cyitoplasm, whereas the envelope proteins remain assaciated with membrane. Since the same ribosomes translate the same mRNA this is very persuasive evidence that neither the ribosome nor the mRNA determines which proteins are synthesised on membrane. In the model described by Wirth et al. it is proposed that at the beginning of translation the 26S RNA is free in the cytoplasm wheneas the core protein is synthesised and released. As translation of the envelope protein proceeds, its amino-terminal sequence initiates an interaction with the membrane leading to the ribosome becoming 'membranebound'. No amino acid data is available for the synthesised proteins but it has been observed that the first of the two glycoproteins is produced in a larger form than is found in the lipid envelope.

The light chain of mouse immunoglobulin has been known for some time to be synthesised in a larger form than is eventually secreted. This has been shown to be due to about 20 extra amino acids preceding the amino terminus of the mature protein. Israel Schechter and his coworkers have been working on the sequence of the amino acids in what they call the 'extra piece' in several diffenent light chains (Burstein \& Schechter Proc. natn. Acad. Sci. U.S.A. 74, 716; 1977). Two completely sequenced, and other partially sequenced, 'extra pieces' reveal that that although these regions ane not particularly homologous in sequence they all contain a very high proportion of hydrophobic amino acids. This fits nicely with the suggestion that this region is responsible for the interaction with endoplasmic reticulum leading to the ribosomes becoming bound to membrane. However, Burstein and Schechter envisage that the hydrophobic region would also allow the protein to associate with other membranes and suggest in particular that it may interact with the cell surface membrane to serve as an antigen-recognising receptor. This hypothesis can be tested experimentally: it will be interesting to see if the 'extra piece' is involved in several different protein-membrane interactions.

\section{Iranian natural history}

\section{from Peter D. Moore}

THE past 30 years have seen an enormous expansion in amateur intenest in natural history in Europe and North America. This surge has been accompanied and encouraged by a parallel proliferation of field guides for the identification of such organisms as birds, plants and butterflies. Taxonomy apart, these developments have been of great benefit to the serious study of distribution patterns, behaviour and migration. In many other parts of the world, where documentation is at present far less thorough, the galvanisation of the potential mass of amatuers may simply be awaiting the provision of accessible field guides to identification.

Peter D. Moore is a lecturer in the Department of Plant Sciences at Kings College London. 